Supporting information:

\title{
Effect of Nanoparticle Synthetic Conditions on Ligand Coating Integrity and Subsequent Nano-Bio Interactions
}

Jessica C. Hsu, ${ }^{1,2}$ Yu Du, ${ }^{3}$ Arjun Sengupta, ${ }^{4}$ Yuxi C. Dong, ${ }^{1,2}$ Katherine J. Mossburg, ${ }^{1,2}$ Mathilde Bouché, ${ }^{1}$ Andrew D. A. Maidment, ${ }^{1}$ Aalim M. Weljie, ${ }^{4}$ and David P. Cormode ${ }^{1,2^{*}}$

1Department of Radiology, University of Pennsylvania, 3400 Spruce St, 1 Silverstein, Philadelphia, PA 19104, USA.

${ }^{2}$ Department of Bioengineering, University of Pennsylvania, Philadelphia, PA, USA.

${ }^{3}$ Division of Gastroenterology and Hepatology, University of Pennsylvania, Philadelphia, PA, USA. ${ }^{4}$ Department of Systems Pharmacology and Translational Therapeutics, University of Pennsylvania, Philadelphia, PA, USA.

${ }^{*}$ Corresponding author

E-mail: david.cormode@pennmedicine.upenn.edu; Tel: 215-615-4656; Fax: 215-662-7868 


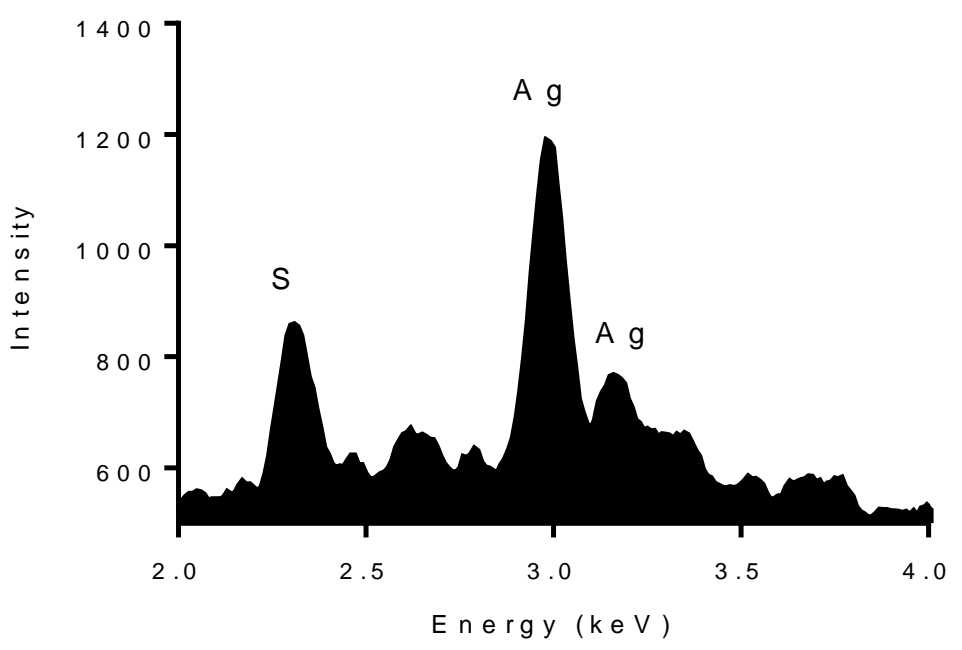

Figure S1 Energy dispersive X-ray spectroscopy spectrum of $3 \mathrm{~nm} \mathrm{Ag} 2 \mathrm{~S}-\mathrm{NP}$ from Synthesis B.
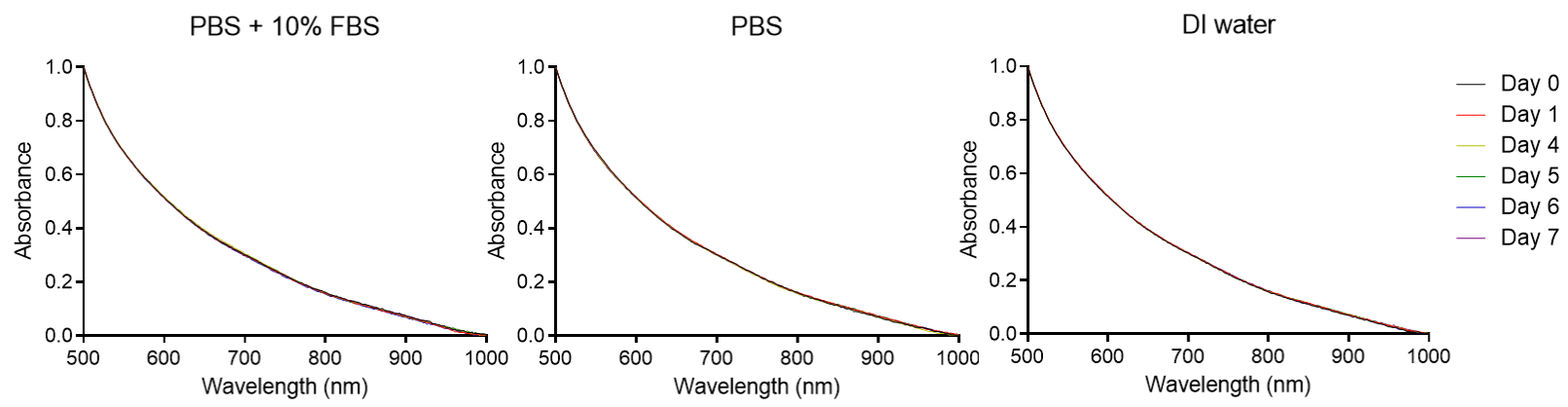

Figure S2 UV-visible spectroscopy measurements of $3 \mathrm{~nm} \mathrm{Ag} 2 \mathrm{~S}-\mathrm{NP}$ from Synthesis B after incubation in DI water, PBS and PBS supplemented with $10 \%$ fetal bovine serum at $37^{\circ} \mathrm{C}$ for 7 days. Some lines may be difficult to visualize as they overlap with other lines. 

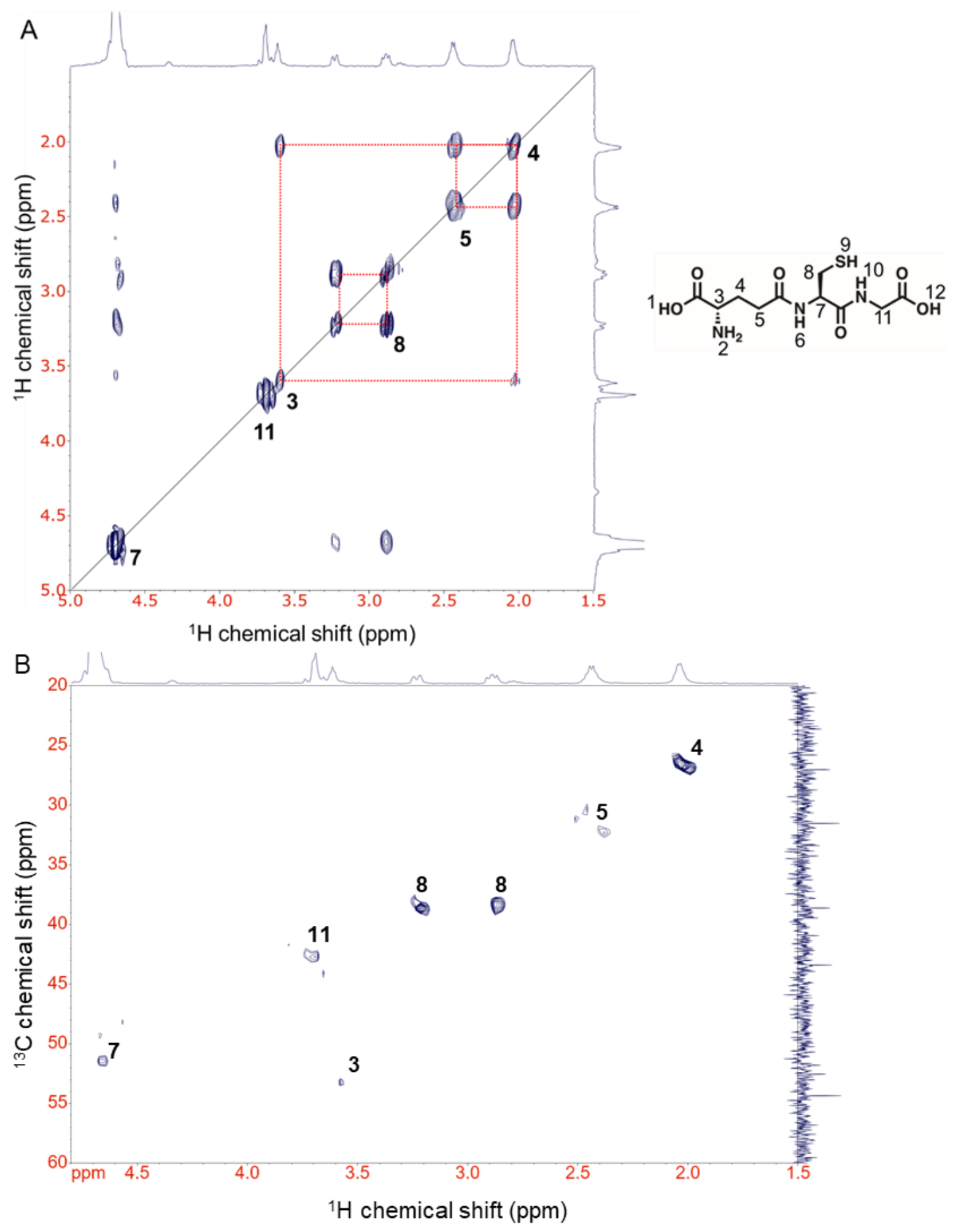

Figure S3 2D NMR spectroscopy of $3 \mathrm{~nm} \mathrm{Ag} 2 \mathrm{~S}-\mathrm{NP}$ synthesized from Synthesis B.

(a) ${ }^{1} \mathrm{H}-{ }^{1} \mathrm{H}$ COSY NMR spectrum and (b) ${ }^{1} \mathrm{H}-{ }^{13} \mathrm{C}$ HSQC NMR spectrum. 


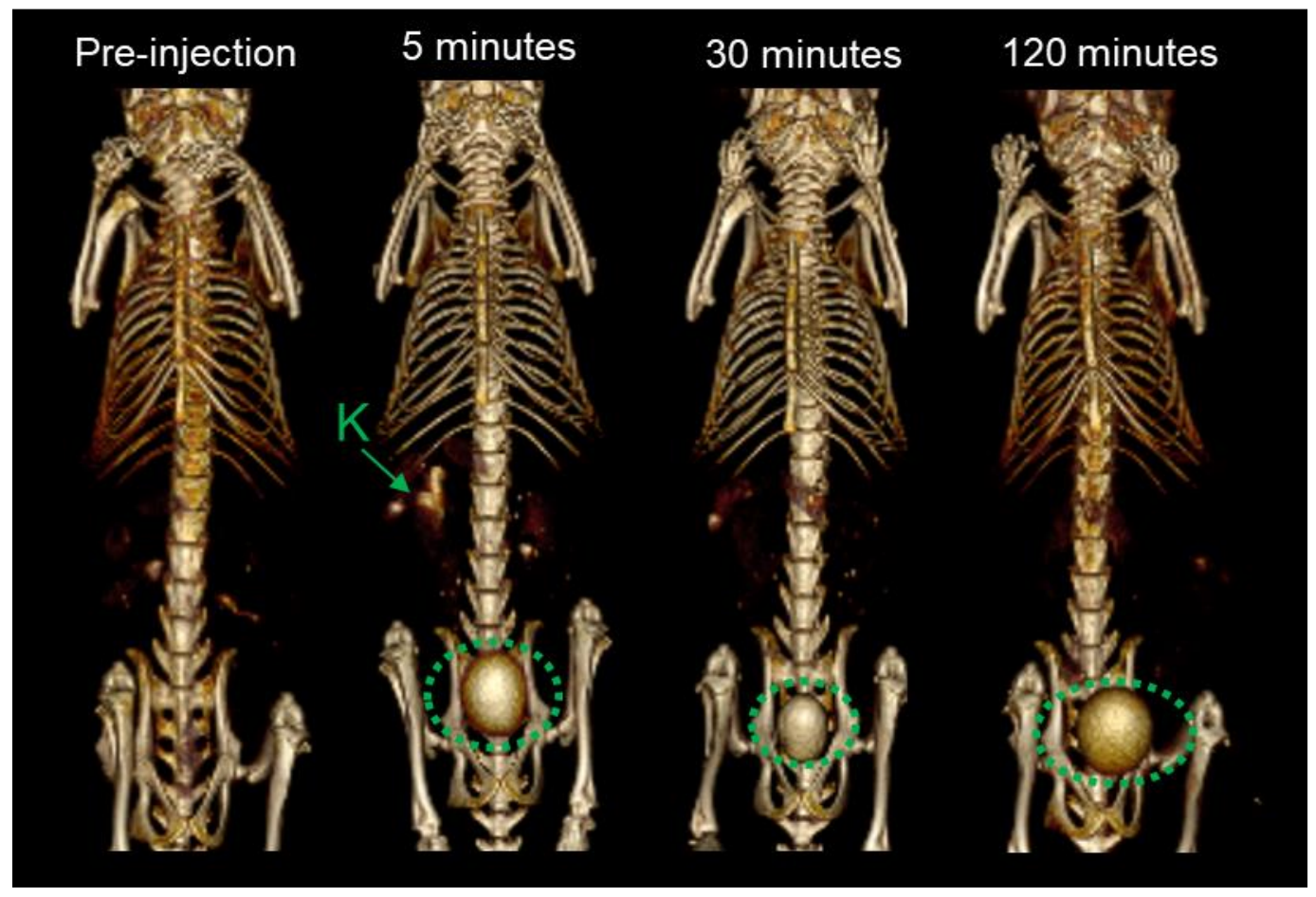

Figure S4 Representative in vivo 3D volume rendered CT images of a mouse injected with $\mathrm{Ag}_{2} \mathrm{~S}$ NP synthesized using high temperature, bulk approach (Synthesis A). The images are shown with a window level of $700 \mathrm{HU}$ and window width of $1000 \mathrm{HU}$. Kidneys are labeled K. Green circles indicate the bladder. 

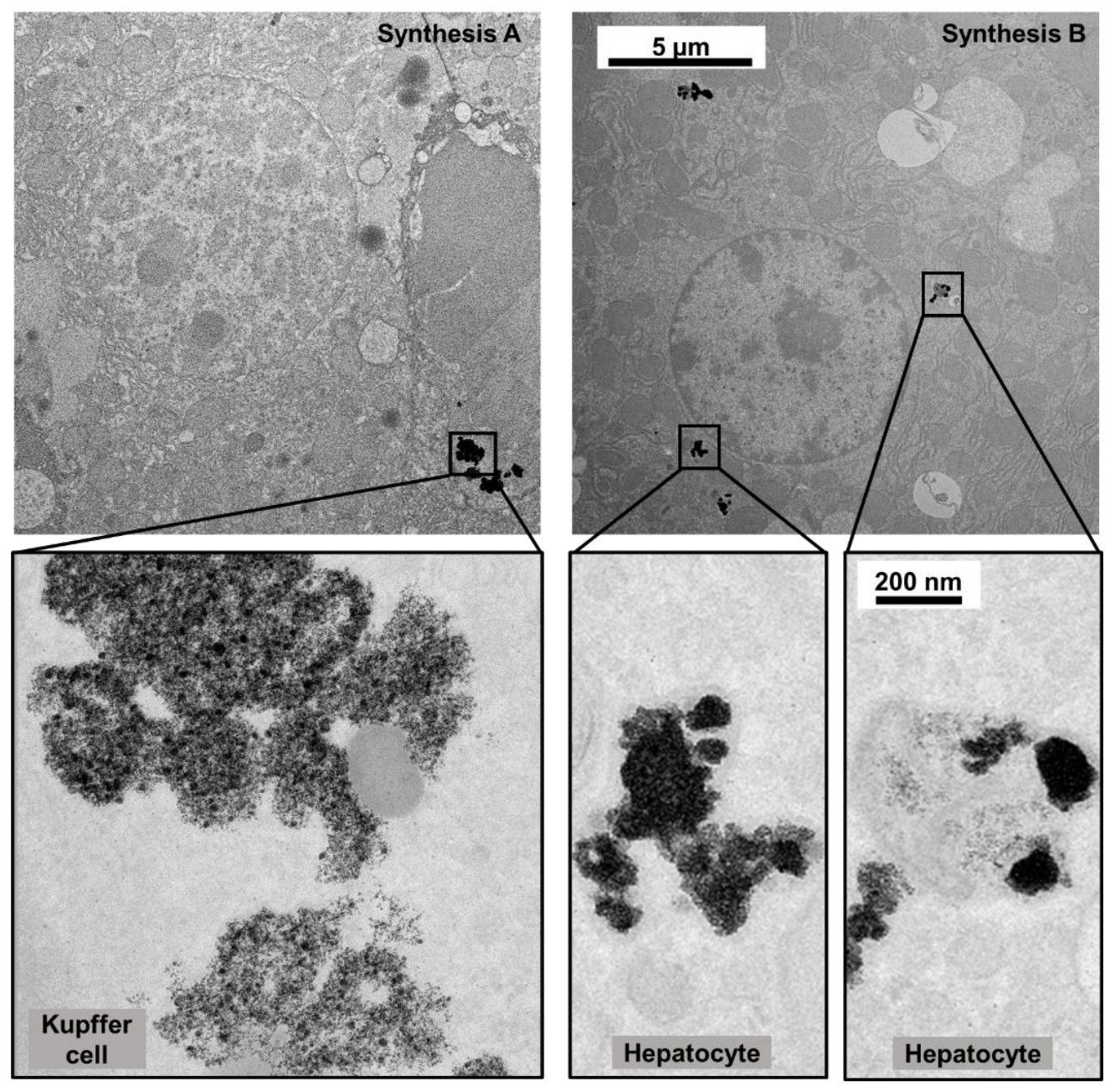

Figure S5 Electron micrographs of liver sections indicating cellular distribution of different $\mathrm{Ag}_{2} \mathrm{~S}$ NP formulations. The scale is indicated in the upper left corner on the rightmost image for each row of images. 

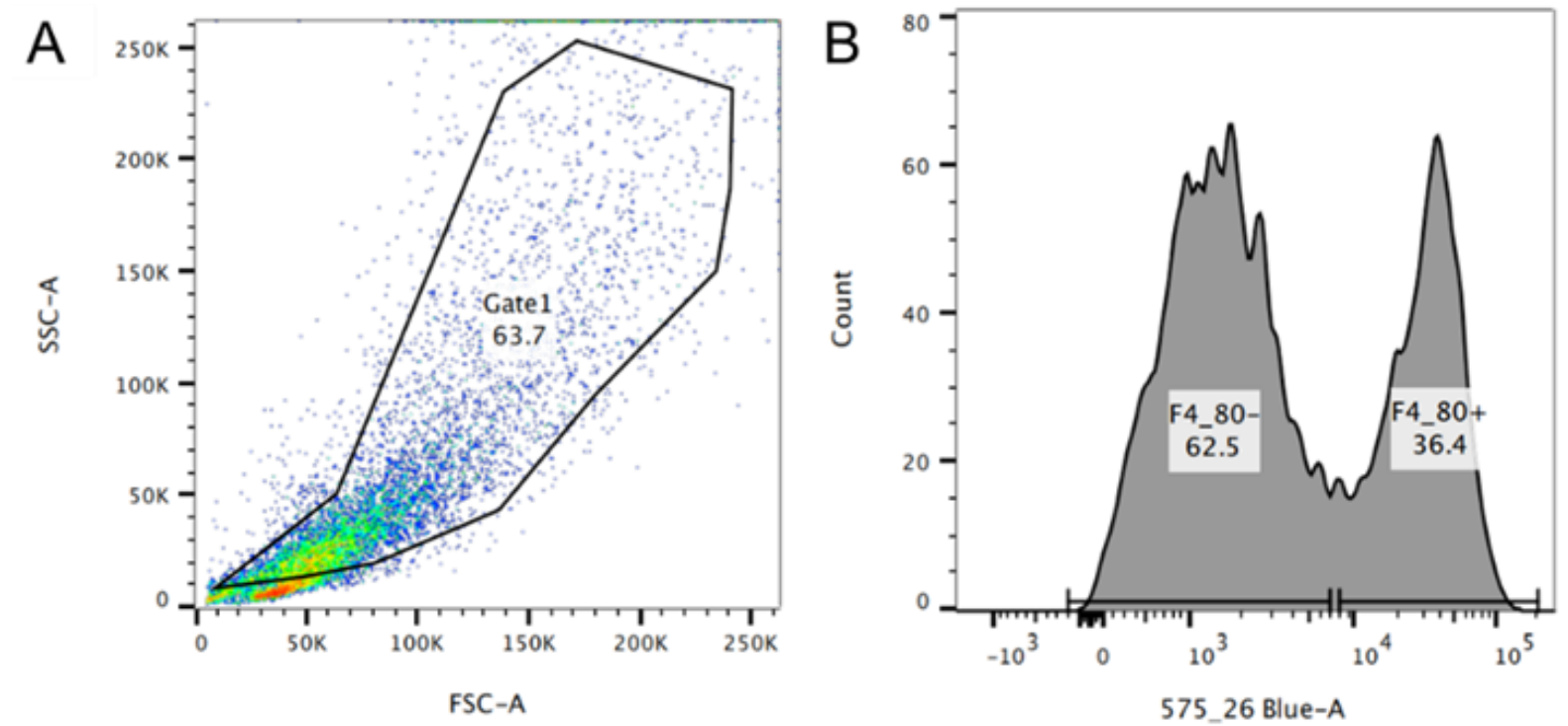

Figure S6 Flow cytometry for primary Kupffer cells.

(a) Representative flow plot with gating to show (b) percentages of F4/80 positive and negative cells in the total nonparenchymal cell population after purification. SSC: side scatter; FSC: forward scatter. 